\title{
LINC00641 inhibits the proliferation and invasion of ovarian cancer cells by targeting miR-320a
}

\author{
Yan $\mathrm{Li}^{1}$, Mengmeng $\mathrm{Lv}^{2}$, Jianhua Wang ${ }^{3}$, Chengzhen Gao ${ }^{1}$ Yuzhong $\mathrm{Wu}^{2}$ \\ ${ }^{1}$ Department of Obstetrics and Gynecology, The Yancheng Clinical College of Xuzhou Medical University \& The First People's Hospital of \\ Yancheng, Yancheng, China; ${ }^{2}$ Department of Gynecologic Oncology, Jiangsu Cancer Hospital \& Jiangsu Institute of Cancer Research \& The \\ Affiliated Cancer Hospital of Nanjing Medical University, Nanjing, China; ${ }^{3}$ Department of Gastroenterology, The Yancheng Clinical College of \\ Xuzhou Medical University \& The First People's Hospital of Yancheng, Yancheng, China. \\ Contributions: (I) Conception and design: Y Li; (II) Administrative support: None; (III) Provision of study materials or patients: J Wang; (IV) \\ Collection and assembly of data: M Lv; (V) Data analysis and interpretation: Y Wu, C Gao; (VI) Manuscript writing: All authors; (VII) Final approval \\ of manuscript: All authors. \\ Correspondence to: Yuzhong Wu. Department of Gynecologic Oncology, Jiangsu Cancer Hospital \& Jiangsu Institute of Cancer Research \& The \\ Affiliated Cancer Hospital of Nanjing Medical University, Nanjing 210009, China. Email: wuyuzhong1127@126.com.
}

Background: Ovarian cancer is a common malignancy of the female reproductive system, with one of the highest mortality rates among all malignant tumors. However, the pathogenesis of ovarian cancer has not been fully elucidated. This study investigated the role and molecular mechanism of LINC00641 in the development and progression of ovarian cancer.

Methods: Quantitative real-time polymerase chain reaction (qRT-PCR) was used to detect the expression level of LINC00641 in ovarian cancer tissue and adjacent normal tissue. Cell Counting Kit-8 (CCK-8), colony formation, and Transwell assays were used to detect the effects of LINC00641 overexpression on the proliferation and migration of ovarian cancer cells. Bioinformatics analysis and luciferase reporter gene assay were employed to detect the binding of LINC00641 to the downstream target molecule, microRNA-320a (miR-320a). Western blotting was used to determine the effect of miR-320a overexpression on the expression of proliferation-related proteins [Ki-67 and proliferating cell nuclear antigen (PCNA)] and invasion-related proteins (E-cadherin, N-cadherin, and vimentin) in overexpressed LINC00641 cells.

Results: qRT-PCR results showed that LINC00641 was under-expressed in ovarian cancer tissue compared to adjacent tissue. Cell function experiments showed that the overexpression of LINC00641 could significantly inhibit the proliferation and migration of ovarian cancer cells. The luciferase reporter gene assay showed that LINC00641 could bind to miR-320a, and the overexpression of LINC00641 could markedly inhibit the expression of miR-320a in ovarian cancer cells. Overexpression of miR-320a could significantly block the inhibitory effect of LINC00641 on the proliferation and migration of ovarian cancer cells.

Conclusions: As a tumor suppressor gene, LINC00641 can inhibit the proliferation and invasion of ovarian cancer cells by targeting miR-320a. The LINC00641/miR-320a axis may be a new target for the early diagnosis, treatment, or prognosis of ovarian cancer patients.

Keywords: LINC00641; miR-320a; ovarian cancer

Submitted Sep 16, 2021. Accepted for publication Nov 17, 2021.

doi: $10.21037 /$ tcr-21-2314

View this article at: https://dx.doi.org/10.21037/tcr-21-2314 


\section{Introduction}

Ovarian cancer is a common malignant tumor of the female reproductive system, and its mortality rate ranks among the highest of all malignant tumors (1). With the continuous progress of medical technology, the treatment of ovarian cancer has been improved to a certain extent; the effective rate of surgical resection combined with chemotherapy for newly treated patients can reach more than $70 \%$ (2). However, the lack of sensitive indicators for the early diagnosis of ovarian cancer coupled with reduced patient sensitivity to chemotherapeutic drugs has led to a 5-year survival rate among ovarian cancer patients of less than $40 \%$ $(3,4)$. Therefore, exploring new molecular markers of the occurrence and development of ovarian cancer is helpful to identify the causes of ovarian tumor tissue, and is of great significance for improving the diagnosis and treatment effect of ovarian cancer and prolonging the life cycle of patients.

Recent studies have shown that long non-coding RNA (lncRNA) is a kind of RNA fragment with a length of more than 200 nucleotides (nt), which does not have the ability to encode protein (5). LncRNA regulates downstream gene expression by regulating chromatin modification and transcription, and participates in many biological processes such as cell proliferation, invasion, and apoptosis $(6,7)$. Numerous studies have shown that lncRNA not only involves the occurrence and progression of a variety of malignant tumors, but can also be used as a diagnostic marker for the early detection of tumors. For example, the expression of LINC00319 is up-regulated in ovarian cancer tissues and cell lines, and the overexpression of LINC00319 significantly promotes the proliferation and invasion of ovarian cancer cells; thus, it can be used as a potential prognostic biomarker for ovarian cancer patients in the future (8). LncRNA pro-transition associated RNA (PTAR) regulates zinc finger Ebox binding homeobox 1 (ZEB1) expression through competitive binding to $\mathrm{miR}-101-3 \mathrm{p}$ to promote epithelial-to-mesenchymal transition (EMT) as well as invasion and metastasis of serous ovarian cancer (9). In addition, studies have shown that the combined detection of plasma lncRNA regulator of reprogramming (ROR) and carbohydrate antigen 125 (CA125) is more valuable in the diagnosis of ovarian cancer than the detection of lncRNA ROR or CA125 alone (10). However, the biological functions of only a few lncRNAs have been verified by experiments so far. Therefore, identifying and clarifying the function of new lncRNAs in ovarian cancer has important potential application prospects for later cancer treatment and diagnosis.

Long intergenic non-protein coding RNA 641 (LINC00641) is a newly identified non-coding RNA (ncRNA), which has been shown to play an important role in the occurrence and development of tumors in recent years. Studies have shown that LINC00641 is involved in the occurrence and progression of a variety of malignant tumors and plays various roles in different malignant tumors. For example, in non-small cell lung cancer, LINC00641 acts as a tumor suppressor gene and is down-regulated in tissues and cells, and LINC00641 overexpression significantly inhibits cell proliferation and induces apoptosis (11). In contrast, in acute myelocytic leukemia (AML), LINC00641 acts as a cancer promoting gene and is highly expressed in AML samples and cell lines; inhibiting LINC00641 significantly inhibits the proliferation, invasion, and cell cycle arrest of AML cells and induces cell apoptosis (12). However, the function and specific molecular mechanism of LINC00641 in ovarian cancer remains unclear. This study aims to investigate the expression of LINC00641 in ovarian cancer, examine the regulatory effect of LINC00641 on the biological function of ovarian cancer cells at the cellular level, and explore its possible regulatory mechanism. We present the following article in accordance with the MDAR reporting checklist (available at https://dx.doi.org/10.21037/tcr-21-2314).

\section{Methods}

\section{Clinical sample collection}

This study was approved by the ethics committee of the First People's Hospital of Yancheng (No. 91921626), and the informed consent was signed by the patients or their families. In total, the tumor tissues and normal ovarian tissues of 24 patients with epithelial ovarian cancer were collected. The age range of the enrolled patients was 36-69 years, with an average age of $54.5 \pm 7.6$ years. The tissue samples of all patients were diagnosed as epithelial ovarian cancer independently by two pathologists. All patients were initial operation patients. The patients did not receive any radiotherapy or other anti-tumor treatment preoperatively. The tissue messenger RNA (mRNA) was extracted and reverse transcribed into complementary DNA (cDNA) for preservation. At the same time, relevant clinical data of patients were collected.

All procedures performed in this study involving human 
participants were in accordance with the Declaration of Helsinki (as revised in 2013).

\section{Cell culture}

Human ovarian cancer cell lines (SKOV-3, OVCA-3, A2780, HO8910, and HEY) and normal ovarian epithelial cell (IOSE80) were purchased from the Cell Center of Chinese Academy of Medical Sciences (Beijing, China). The cells were cultured in Roswell Park Memorial Institute-1640 (RPMI-1640) medium (10\% fetal bovine serum) supplemented with $100 \mathrm{U} / \mathrm{mL}$ penicillin and $100 \mathrm{mg} / \mathrm{mL}$ streptomycin. The cells were cultured in a cell incubator with $5 \% \mathrm{CO}_{2}$ and $37^{\circ} \mathrm{C}$ saturated humidity, and the cell culture medium was replaced every $2-3$ days. The cells were sub-cultured at a rate of 1:2-1:3 based on cell growth conditions. The cells in the logarithmic growth phase were used for subsequent experiments and mRNA extraction.

\section{Real-time polymerase chain reaction (RT-PCR) assay}

The total RNA of the cells was extracted using Trizol reagent (Source Leaf Biology Co., LTD, Shanghai, China). Ovarian cancer cells in the logarithmic phase were taken. One-milliliter Trizol reagent was added to the cells, blown evenly with a pipette, and placed on ice for 5 min to completely separate the nucleoprotein complex. The content and purity of the RNA were determined by NanoDrop spectrophotometer (Thermo Fisher Scientific Inc. USA). RNA purity was qualified when the OD260 nm/ OD280 $\mathrm{nm}$ value was ranged between 1.8 and 2.0.

Quantitative real-time polymerase chain reaction (qRTPCR) was performed using a two-step method. The reaction conditions were as follows: pre-denaturation at $95{ }^{\circ} \mathrm{C}$ for $30 \mathrm{~s} ; 40$ cycles of denaturation at $95.0^{\circ} \mathrm{C}$ for $5 \mathrm{~s}$ and annealing and elongation at $60.0^{\circ} \mathrm{C}$ for $30 \mathrm{~s}$; and $72{ }^{\circ} \mathrm{C}$ for $30 \mathrm{~s}$. After the reaction, the dissolution curve was generated by the built-in PCR amplifier software. The relative expression of the target gene in each group was analyzed using the $2^{-\Delta \Delta \mathrm{Ct}}$ method, where $\Delta \Delta \mathrm{Ct}=\Delta \mathrm{Ct}$ (experimental group) $-\Delta \mathrm{Ct}$ (control group), $\Delta \mathrm{Ct}=\mathrm{Ct}$ (target gene) - $\Delta \mathrm{Ct}$ [glyceraldehyde-3-phosphate dehydrogenase (GAPDH)], and $\mathrm{Ct}$ is the number of amplification cycles required for the fluorescence intensity to reach the threshold.

* LINC00641: forward, 5'-GTAACTCTATGTACA ACGTTAA-3'; reverse, 5'-TAGAAGTCAACTCAT

\author{
TATGCTGCTG-3'; \\ * GaPdH: forward, 5'-GGaATCCA \\ C T G G C G T C T T C A - 3 '; r e ver s e : \\ 5'-GGTTCACGCCCATCACAAAC-3'; \\ * miR-320a: forward, 5'- GTTGGATCCGGCGTT \\ TCCTTCCGACATG-3'; reverse, 5'-GCTGAATT \\ CGTCCACTGCGGCTGTTC-3'; \\ * U6: forward, 5'-CTCGCTTCGGCAGCACA-3'; \\ reverse: 5'-AACGCTTCACGAATTTGCGT-3'.
}

\section{Transfection assay}

The plasmids [pcDNA-641 and its empty vector (pcDNAcontrol), miR-320a mimics and its empty vector (control mimics)] were synthesized by Sangon Biotech (Shanghai, China). Ovarian cancer cells in the exponential growth stage were collected. After counting, the cells were seeded and inoculated in a six-well plate at a density of $5 \times 10^{5}$ cells per well, and cultured in an incubator at $37^{\circ} \mathrm{C}$ with $5 \% \mathrm{CO}_{2}$. Transfection solution-1 was a mixture of $8 \mu \mathrm{L}$ Lipofectamine 2000 and $100 \mu \mathrm{L}$ RPMI-1640 medium, while transfection solution-2 was a mixture of $2 \mu \mathrm{g}$ plasmid and $100 \mu \mathrm{L}$ RPMI-1640 medium. After the preparation of transfection solutions 1 and 2, they were incubated at room temperature for $5 \mathrm{~min}$, mixed, and then incubated at room temperature for $20 \mathrm{~min}$. The old medium supernatant was discarded, and RPMI-1640 complete medium was added. Next, the cells were placed in a $37^{\circ} \mathrm{C}$ incubator with $5 \%$ $\mathrm{CO}_{2}$ for further culturing. After 4-6 h, the transfection mixture was removed and replaced with fresh Dulbecco's Modified Eagle Medium (DMEM) medium, and the culture plate was subsequently placed in the incubator for $48 \mathrm{~h}$.

\section{Cell Counting Kit-8 assay (CCK-8)}

The cell proliferation activity of each group was detected by CCK-8. Cells in good condition were taken and washed twice with prechilled phosphate buffer saline (PBS) buffer. The cells were digested with $0.25 \%$ trypsin and made into single-cell suspension. After counting and adjusting the density, the cells were inoculated into a 96-well plate at a density of $5 \times 10^{3}$ cells per well. Each well was set in quintuplicate, and $100 \mu \mathrm{L}$ medium was added into each well. When the specified time was reached $(1,2,3$, and 4 days), $10 \mu \mathrm{L}$ CCK-8 solution mixed with $100 \mu \mathrm{L}$ medium was added into the 96-well plate and cultured for $2 \mathrm{~h}$ at $37{ }^{\circ} \mathrm{C}$ in dark. The procedure was performed in strict accordance with the instructions of the kit. The absorbance 
(OD) at $450 \mathrm{~nm}$ was then measured in a microplate reader and the average value was taken. The cell growth curve of each group of cells was drawn based on the results.

\section{Colony formation assay}

Cells in good condition were taken and washed twice with prechilled PBS buffer. The cells were digested with trypsin and made into a single-cell suspension. After counting, the cells were seeded into a 96-well plate at a density of 400 cells per well. The six-well plate was also prewashed with prechilled PBS buffer. The plate was shaken gently to disperse the cells evenly, and then placed in a $37{ }^{\circ} \mathrm{C}$ incubator with $5 \% \mathrm{CO}_{2}$. The cell growth was observed on a regular basis and the medium was replaced on time. When visible cell colonies appeared, the culture was stopped. The original culture medium was discarded, and the cells were washed twice with PBS buffer, immersed with an appropriate amount of paraformaldehyde solution, fixed for $15 \mathrm{~min}$, and stained with $4 \mathrm{~mL}$ of crystal violet. Next, the dye solution was recovered, and the six-well plate was washed with PBS buffer to remove the excess dye solution. After drying at room temperature, the plate was observed under a microscope, and the cell images were obtained for counting and analysis.

\section{Transwell assay}

Cells in good condition were taken and washed twice with prechilled PBS buffer. The cells were digested with trypsin and made into a single-cell suspension with serum free medium. The cells were then transferred to a $15-\mathrm{mL}$ centrifuge tube for counting. The upper chamber of the Transwell was added with $200 \mu \mathrm{L}$ of cell suspension $\left(2 \times 10^{5}\right.$ cells per well) and serum-free DMEM. Each well was set in triplicate, and bubbles were avoided during the procedure. The Transwells were placed in incubator for further culturing. After $36 \mathrm{~h}$ (different culture time for different cells), the culture was stopped, the chamber was removed, and the supernatant and residual liquid was discarded. The plate was washed with PBS buffer three times, and the Matrigel in the upper chamber was removed using a cotton swab. The chambers were placed in a 24 -well plate containing $700 \mu \mathrm{L}$ methanol for $0.5 \mathrm{~h}$. After fixation, the chambers were cleaned with PBS buffer three times. Crystal violet $(0.1 \%)$ was added and dyed for approximately $30 \mathrm{~min}$ to ensure that the crystal violet dye solution could soak into the bottom of the chamber. The chambers were washed with PBS buffer three times to clean the residual crystal violet dye as much as possible, and the cells were observed under an inverted microscope (Shenzhen Xingming Optical Instrument Co., Ltd., China). The number of cells stained with crystal violet in five visual fields was calculated.

\section{Luciferase reporter gene assay}

Firstly, the target genes of LINC00641 were predicted by bioinformatics analysis. It was found that LINC00641 may regulate downstream miR-320a. Next, a dual luciferase assay was used to verify the targeting relationship between LINC00641 and miR-320a. The specific steps were as follows. The LINC00641 RNA with 3'-untranslated region (3'-UTR) was amplified, and the amplified fragment was inserted into the pGL3-promoter vector and co-transfected with the PRL-SV40 plasmid expressing renin fluorescence. The wild-type (Wt) plasmid was pGL3-Wt-LINC00641 3 'UTR (LINC00641-Wt), and the mutant (Mut) plasmid was pGL3-Mut-LINC00641 3'UTR (LINC00641Mut). The Wt plasmid and Mut plasmid were added to $50 \mu \mathrm{L}$ of serum-free medium with miR-320a mimics and mimics control, respectively. After mixing, the luciferase recombinant vector was added at a ratio of $1: 2$. Dual luciferase detection was then performed using the dualluciferase reporter assay system.

\section{Western blot}

Cells in a good growth state were collected and added with pre-cooled $100 \mu \mathrm{L}$ radioimmunoprecipitation assay (RIPA) lysis buffer. The culture bottle was placed on a $4{ }^{\circ} \mathrm{C}$ shaking table and gently shaken for $0.5 \mathrm{~h}$. The cell suspension in the culture bottle was then transferred to a $2.5-\mathrm{mL}$ centrifuge tube and centrifuged at $14,000 \mathrm{rpm}$ at $4{ }^{\circ} \mathrm{C}$ for $10 \mathrm{~min}$. The supernatant was transferred to a new centrifuge tube, sub packed, and stored in a refrigerator at $-80^{\circ} \mathrm{C}$. The Bradford method was used to detect the protein concentration of the extracted total protein sample. Separation gel and concentrated gel were prepared using the conventional method. The samples were added into gel lanes with 60-80 per lane. Electrophoresis was performed using a constant voltage of $40 \mathrm{~V}$ (concentrated gel)/100 V (separation gel), until the bromophenol blue reached the edge of the gel. A polyvinylidene fluoride (PVDF) membrane was blocked with $5 \%$ skim milk powder at room temperature for $2 \mathrm{~h}$, and the primary antibodies Ki-67 (1:1,000), GAPDH (1:1,000; internal reference), 
proliferating cell nuclear antigen (PCNA) (1:800), E-cadherin (1:800), $\mathrm{N}$-cadherin $(1: 1,000)$, and vimentin $(1: 1,000)$ diluted with $5 \%$ bovine serum albumin (BSA) were added respectively for incubation overnight at $4{ }^{\circ} \mathrm{C}$. The membrane was washed four times with tris-buffered saline Tween (TBST), added with a secondary antibody $(1: 5,000)$ diluted with $5 \%$ BSA, and incubated at room temperature for $1.5 \mathrm{~h}$. The membrane was washed four times again with TBST for 5 min each time, keeping the protein side downward. Liquid $\mathrm{A}$ and liquid $\mathrm{B}$ of electrochemiluminescence (ECL) developer were mixed in equal volumes and dropped into the PVDF membrane for development. Photographs were taken with the Tanon 5200 (Shenzhen Xingming Optical Instrument Co., Ltd., China).

\section{Statistical analysis}

The data were analyzed using SPSS 19.0. Measurement data were shown as mean \pm standard deviation (mean \pm $\mathrm{SD}$ ); measurement data with a normal distribution were compared using the $t$-test or analysis of variance (ANOVA), while those did not follow a normal distribution were tested using the non-parametric rank sum test. The Chi-square test was used for counting data comparation. $\mathrm{P}<0.05$ was considered statistically significant.

\section{Results}

\section{LINC00641 is under-expressed in ovarian cancer tissues and cell lines}

The expression of LINC00641 in ovarian cancer tissues was analyzed using The Cancer Genome Atlas (TCGA) database. The results showed that LINC00641 was underexpressed in ovarian cancer tissues compared to adjacent tissues (Figure 1A). Further analysis showed that the expression of LINC00641 decreased with the increase of pathological grade (Figure 1B). Additionally, the expression of LINC00641 in 24 pairs of ovarian cancer tissues and adjacent tissues was detected by qRT-PCR. The detection results were consistent with the TCGA database analysis; that is, LINC00641 was under-expressed in ovarian cancer tissues (Figure 1C). It was found that the expression level of LINC00641 in ovarian cancer cells was significantly lower than that in normal ovarian epithelial cells IOSE80, and the expression level in SKOV-3 and HEY cells was the lowest (Figure 1D). Therefore, ovarian cancer cell lines SKOV-3 and HEY were selected for subsequent functional experiments and molecular mechanism studies.

\section{Overexpression of LINC00641 inbibits the proliferative and migrative capacity of ovarian cancer cells}

To further study the function of LINC00641 in the occurrence and development of ovarian cancer, the LINC00641 overexpression vector (pcDNA-641) and control vector (pcDNA-control) were constructed first, which were then transfected into SKOV-3 and HEY cells to detect the overexpression efficiency of LINC00641. The results showed that compared to the control vector group (pcDNA-control), transfection of pcDNA-641 significantly increased the expression level of LINC00641 in ovarian cancer cells (SKOV-3 and HEY) (Figure 2A). Subsequently, the effect of LINC00641 overexpression on the proliferation of SKOV-3 and HEY in ovarian cancer cells was detected by CCK- 8 . The results showed that compared to the control vector group (pcDNA-control), overexpression of LINC00641 significantly inhibited the proliferation of ovarian cancer cells (SKOV-3 and HEY) (Figure 2B). The colony formation assay showed that compared to the control vector group (pcDNA-control), overexpression of LINC00641 significantly inhibited the colony formation ability of ovarian cancer cells (SKOV3 and HEY) (Figure 2C). In addition, the effect of LINC00641 expression on the migrative ability of ovarian cancer cells was further detected by Transwell assay. The results showed that, compared to the control vector group (pcDNA-control), overexpression of LINC00641 markedly inhibited the migrative ability of ovarian cancer cells (SKOV-3 and HEY) (Figure 2D). These results indicated that LINC00641overexpression significantly repressed the proliferation and migration of ovarian cancer cells.

\section{LINC00641 directly regulates miR-320a expression in ovarian cancer cells}

The bioinformatics online prediction software (Starbase, Guangdong, China, https://starbase.sysu.edu.cn/starbase2/ index.php) found that LINC00641 sequence contains the binding site with miR-320a (Figure $3 A$ ). Based on the predicted LINC00641 binding sequence with miR-320a, the vector containing the normal sequence (LINC00641-Wt) and the vector containing the mutant base (LINC00641Mut) were constructed. These were respectively cotransfected into the cells with blank control group (control mimics) and miR-320a mimics to detect luciferase 
A

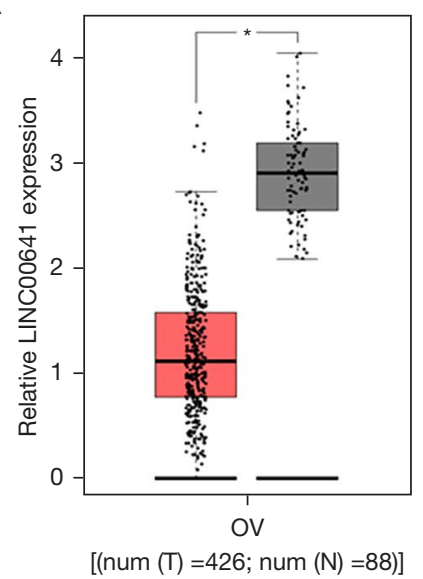

C

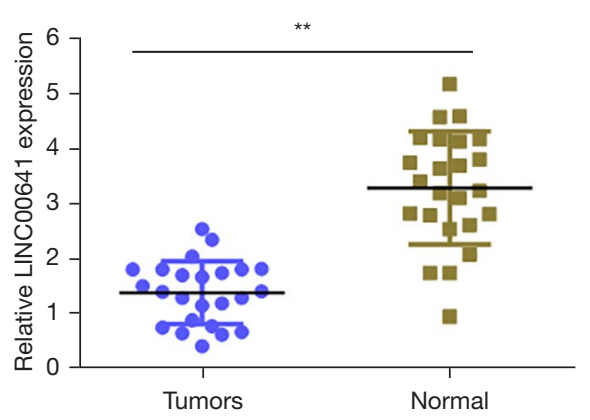

B

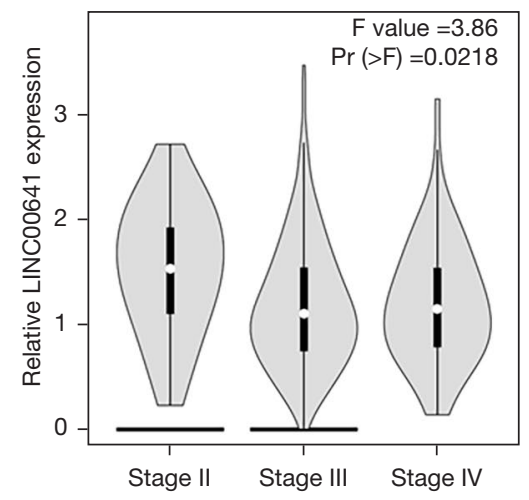

D

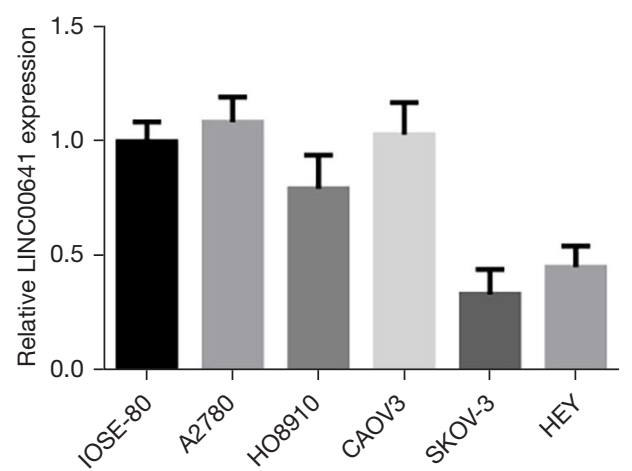

Figure 1 LINC00641 was under-expressed in ovarian cancer tissues and cell lines. (A) LINC00641 expression in ovarian cancer tissue and adjacent tissue analyzed using TCGA database; (B) correlation between LINC00641 expression and pathological grade of ovarian cancer; (C) LINC00641 expression in 24 ovarian cancer patients and paired adjacent tissue samples detected by qRT-PCR; (D) LINC00641 expression in ovarian cancer cell lines and normal gastric fibroblasts detected by qRT-PCR. *, $\mathrm{P}<0.05$; **, $\mathrm{P}<0.01$. TCGA, The Cancer Genome Atlas.

activity. The results showed that transfection of miR-320a mimics and the vector containing the normal sequence (LINC00641-Wt) significantly inhibited the luciferase activity of cells, while the mutant type (LINC00641-Mut) did not have a notable effect (Figure 3B). qRT-PCR was also used to detect the effect of LINC00641 overexpression on the expression of miR-320a. The results showed that compared to the control vector group (pcDNA-control), the expression level of miR-320a was increased in LINC00641overexpressing ovarian cancer cells (SKOV-3 and HEY) (Figure 3C). In addition, the expression level of miR-320a in ovarian cancer tissue and para-cancer tissue was further detected, and the results showed that miR-320a was highly expressed in ovarian cancer tissue compared to paracancer tissue (Figure 3D). The above results showed that LINC00641 could directly regulate the expression of miR320a in ovarian cancer cells.

\section{LINC00641 regulates the proliferation and migration of ovarian cancer cells by targeting $m i R-320 a$}

To further determine the effect of LINC00641 on the proliferative and migrative ability of ovarian cancer cells (SKOV-3) by targeting miR-320a expression, miR-320a mimics were transfected into overexpressed LINC00641 ovarian cancer cells, and cell activity was then detected by CCK-8. The results showed that LINC00641 overexpression significantly inhibited the proliferation of ovarian cancer cells (SKOV-3), while miR-320a overexpression (miR-320a mimics) markedly blocked the inhibitory effect of LINC00641 overexpression on the proliferation of ovarian cancer cells (Figure 4A).

The expression levels of proliferation-related proteins (Ki-67 and PCNA) were detected by western blot, and the results showed that LINC00641 overexpression 

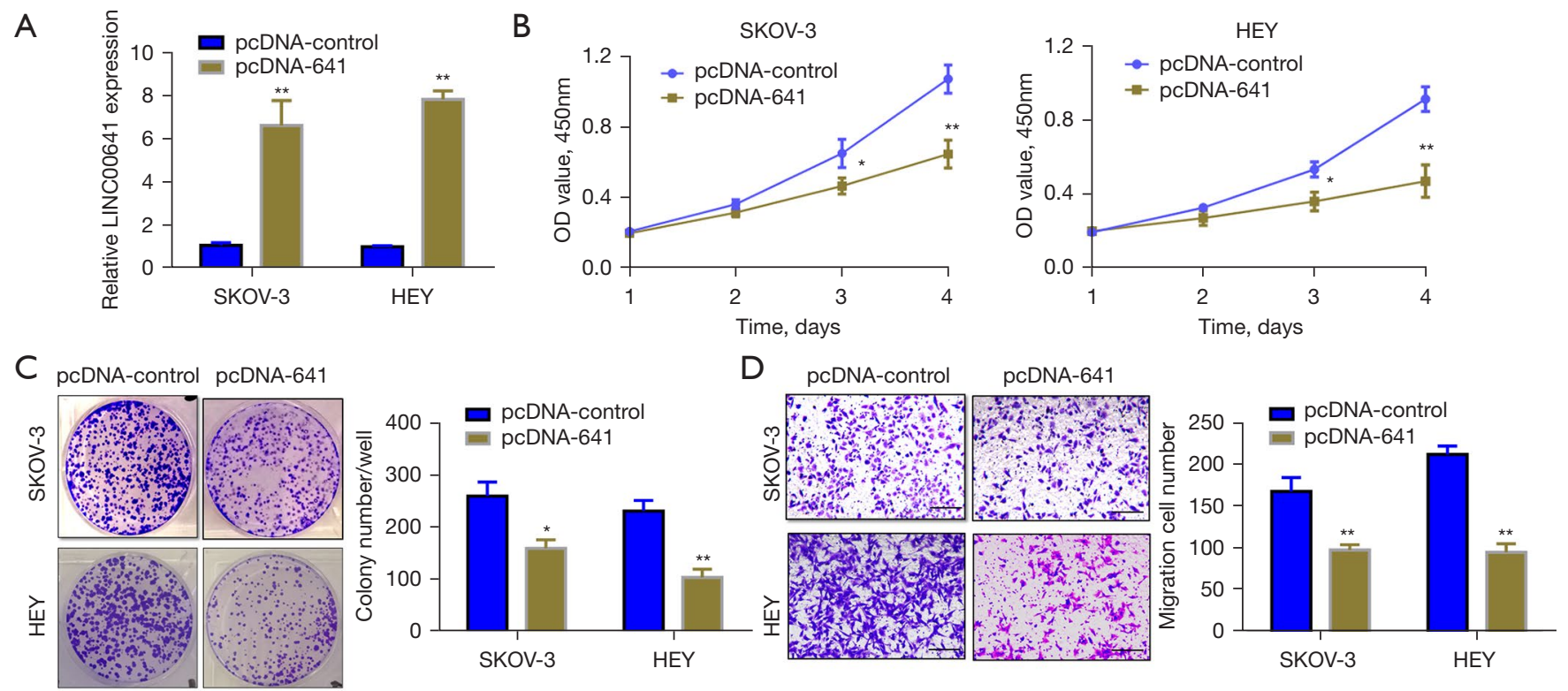

Figure 2 LINC00641 overexpression significantly repressed the proliferation and migration of ovarian cancer cells. (A) qRT-PCR detected LINC00641 overexpression in ovarian cancer cells; (B) CCK-8 detected the effect of LINC00641 overexpression on the proliferation of ovarian cancer cells; (C) colony formation assay detected the effect of LINC00641 overexpression on the colony formation ability of ovarian cancer cells (crystal violet staining); (D) Transwell assay detected the effect of LINC00641 overexpression on the migrative ability of ovarian cancer cells (crystal violet staining). Scale bar: $200 \mu \mathrm{m}$. *, $\mathrm{P}<0.05$; **, $\mathrm{P}<0.01$. qRT-PCR, quantitative real-time polymerase chain reaction.
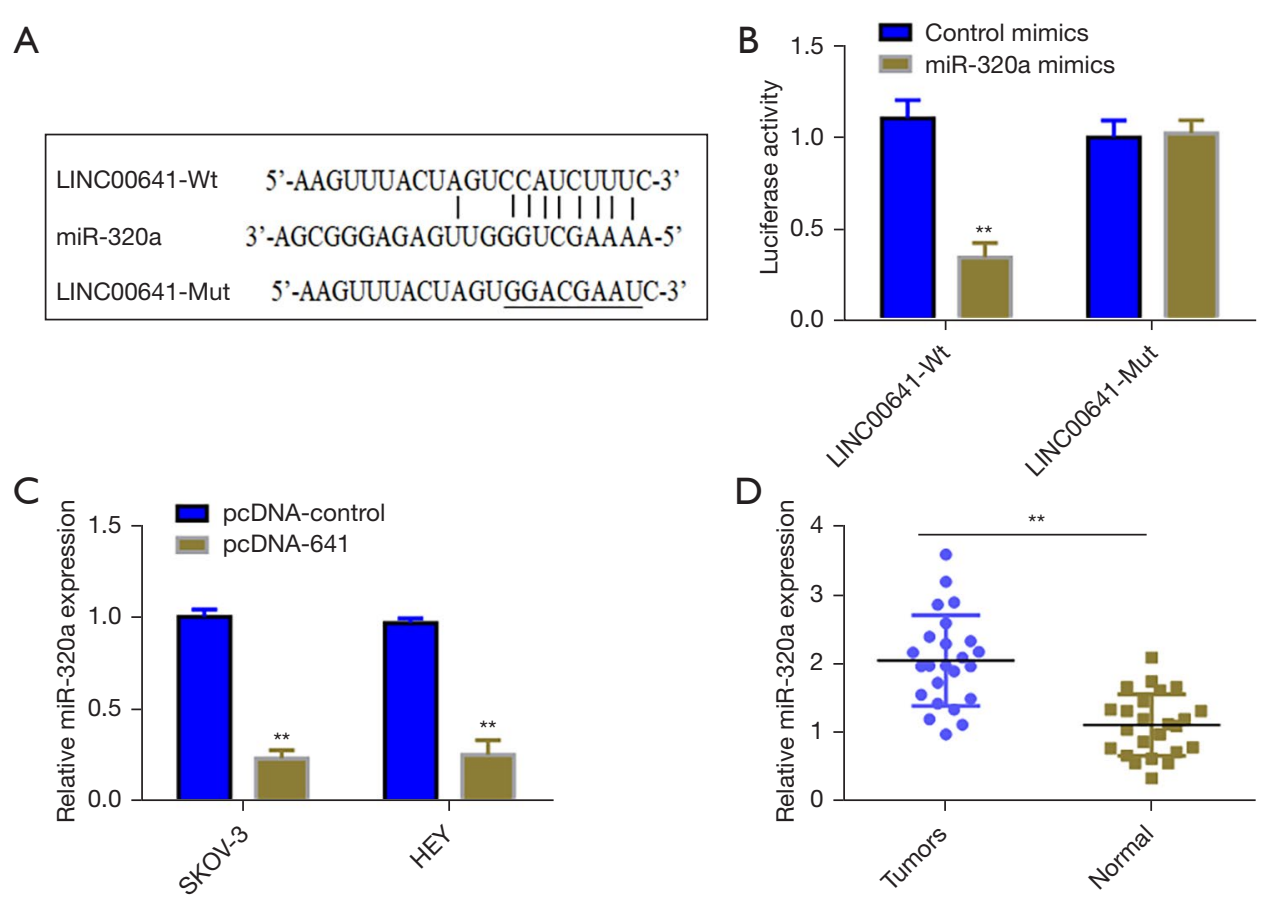

Figure 3 LINC00641 targets miR-320a expression. (A) Prediction of regional binding sites between LINC00641 and mir-320a; (B) the luciferase reporter gene detected the binding of LINC00641 to miR-320a; (C) qRT-PCR detected the effect of LINC00641 overexpression on miR-320a expression level; (D) qRT-PCR detected miR-320a expression in 24 ovarian cancer patients and paired adjacent tissue samples. ${ }^{*}, \mathrm{P}<0.05 ;{ }^{* *}, \mathrm{P}<0.01$. qRT-PCR, quantitative real-time polymerase chain reaction. 
A

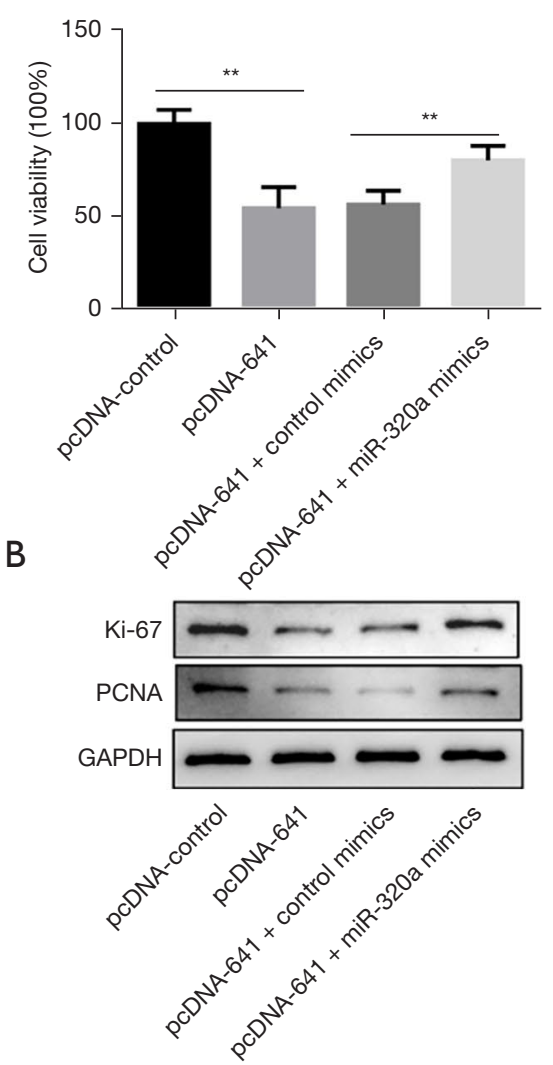

C

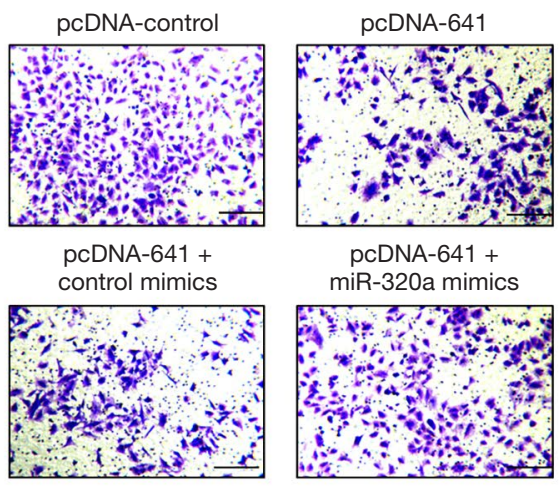

D

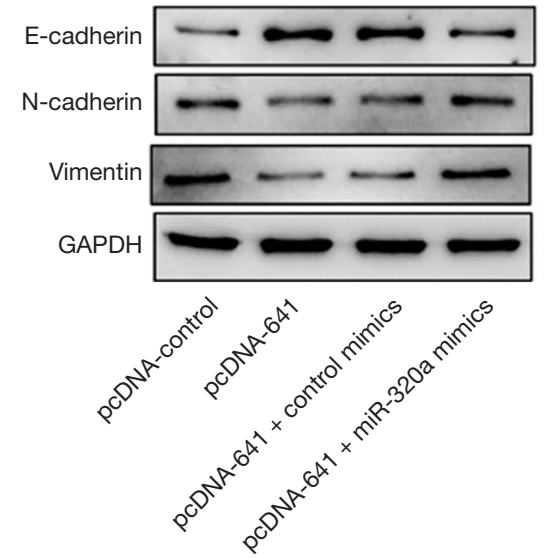

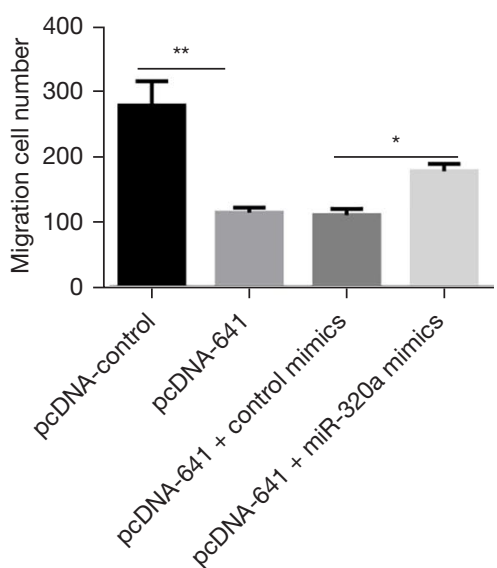

Figure 4 LINC00641 regulates the proliferation and migration of ovarian cancer cells (SKOV-3) by targeting miR-320a expression. (A) CCK-8 detected the effect of miR-320a overexpression on the proliferation of ovarian cancer cells that overexpressed LINC00641; (B) Western blot detected the effect miR-320a overexpression on the expression of Ki-67 and PCNA in ovarian cancer cells that overexpressed LINC00641; (C) Transwell detected the effect of miR-320a overexpression on migration of ovarian cancer cells overexpressing LINC00641 (crystal violet staining, scale bar: $200 \mu \mathrm{m}$ ); (D) Western blot detected the effect of miR-320a overexpression on the expression of E-cadherin, $\mathrm{N}$-cadherin, and vimentin in ovarian cancer cells that overexpressed LINC00641. * $\mathrm{P}<0.05 ;{ }^{* *}, \mathrm{P}<0.01$. GAPDH, lyceraldehyde-3phosphate dehydrogenase; PCNA, proliferating cell nuclear antigen.

significantly inhibited the expression levels of Ki-67 and PCNA in ovarian cancer cells (SKOV-3), while miR-320a overexpression (miR-320a mimics) notably blocked the inhibitory effect of LINC00641 overexpression on the expression of Ki-67 and PCNA proteins in ovarian cancer cells (Figure 4B). In addition, the Transwell assay showed that LINC00641 overexpression significantly inhibited the migration of ovarian cancer cells (SKOV-3), and miR320a overexpression (miR-320a mimics) substantially blocked the inhibitory effect of LINC00641 overexpression on the migration of ovarian cancer cells (Figure 4C). The expression levels of migration-related proteins (E-cadherin, $\mathrm{N}$-cadherin, and vimentin) were further detected by western blot. The results showed that LINC00641 overexpression significantly promoted the expression of E-cadherin and inhibited the expression of $\mathrm{N}$-cadherin and vimentin; meanwhile, miR-320a overexpression (miR-320a mimics) markedly blocked the up-regulation of E-cadherin expression by LINC00641 overexpression and inhibited the expression levels of $\mathrm{N}$-cadherin and vimentin (Figure $4 D$ ). The above experimental results indicated that LINC00641 can regulate the proliferation and invasion of ovarian cancer cells by targeting miR-320a.

\section{Discussion}

Ovarian cancer is among the most common malignant tumors of the female reproductive system. It ranks third 
(after cervical cancer and endometrial cancer) in terms of incidence, and this trend is increasing every year; however, it has the highest mortality rate (1). Therefore, further elucidation of the formation and metastasis mechanisms of ovarian cancer as well as improving the early diagnosis, targeted therapy, and prognosis of ovarian cancer is the focus of future research. A large number of recent studies have shown that the maladjustment of lncRNAs is closely associated to the occurrence and progression of tumors, which indicates that lncRNAs are expected to become new biomarkers for the early diagnosis, prognosis, and treatment of tumors $(6,13)$. The present study showed that LINC00641 was lowly expressed in ovarian cancer and significantly correlated with the TNM stage of ovarian cancer patients. In vitro function experiments showed that LINC00641 overexpression markedly suppressed the proliferation and migration of ovarian cancer cells. Molecular mechanism studies showed that LINC00641 could bind miR-320a, and miR-320a overexpression could notably block the inhibitory effect of LINC00641 overexpression on the proliferation and migration of ovarian cancer cells.

LncRNAs are a class of endogenous ncRNAs with a length of more than 200 nucleotides, accounting for $70 \%$ of ncRNAs (14). They were previously considered insignificant, but in recent years, an increasing number of studies have shown that IncRNA is closely associated to the occurrence and development of human diseases, especially the cell processes related to tumor proliferation, differentiation, invasion, and metastasis (15). For example, lncRNA metastasis-associated lung adenocarcinoma transcript 1 (MALAT1) promotes the proliferation and metastasis of ovarian cancer cells via the phosphoinositide 3-kinase (PI3K)/ protein kinase B (AKT) signaling pathway (16). LncRNA TP73-AS1 promotes the proliferation and metastasis of ovarian cancer cells by regulating the expression of matrix metalloproteinase 2 (MMP2) and matrix metalloproteinase 9 (MMP9) (17). In this study, it was found that LINC00641 was lowly expressed in ovarian cancer and was closely related to the TNM stage of ovarian cancer patients. Cell function experiments showed that LINC00641 overexpression could significantly inhibit the proliferative and migrative capacity of ovarian cancer cells. Based on the above experimental results, it is inferred that LINC00641 is a tumor suppressor gene in ovarian cancer. However, the underlying molecular mechanism of LINC00641's biological function remains unclear.

Previous studies have shown that lncRNA has no ability to encode proteins, but is involved in the regulation of protein encoding genes at various levels (epigenetic, transcriptional, and post-transcriptional levels) in the form of RNA $(18,19)$. For example, LINC00641 mainly affects the expression of downstream target genes by regulating the expression of intermediate miRNA; that is, binding miRNA like a sponge, so as to prevent the binding of miRNA with its target molecule mRNA. In gastric cancer, LINC00641 activates oxaliplatin resistance in gastric cancer cells by regulating the expression of miR-582-5p (20). LINC00641 inhibits the proliferation and invasion of glioma cells by targeting the miR-4262/recombinant neurogranin (NRGN) axis, which provides a new potential therapeutic target for the treatment of glioma (21). The present study confirmed that LINC00641 can bind miR-320a to regulate its expression through bioinformatics and luciferase reporter gene; that is, LINC00641 overexpression significantly reduced the expression of miR-320a in ovarian cancer cells. Studies have shown that miR-320a plays an important regulatory role in a variety of tumors as a tumor suppressor or promoter. For example, miR-320a is lowly expressed in melanoma tissues and cells, and miR-320a overexpression can markedly inhibit cell proliferation and migration (22). In gastric cancer, miR-320a inhibits the proliferation of gastric cancer cells by targeting RAB14 (23). However, miR$320 \mathrm{a}$ is highly expressed in retinoblastoma cell lines, and significantly inhibits cell proliferation and migration (24). Another study showed that miR-320a is highly expressed in ovarian cancer, and miR-320a overexpression significantly blocks the inhibitory effect of LINC00641 overexpression on the proliferation and migration of ovarian cancer cells (23). The results of the present study are consistent with the previous research. Since miRNA does not encode protein and participates in biological function by regulating the expression of target genes, the downstream genes of miR-320a will be explored in subsequent studies.

In conclusion, LINC00641 was lowly expressed in ovarian cancer and was closely related to the TNM stage. LINC00641 overexpression could significantly inhibit the proliferative and migrative capacity of ovarian cancer cells. Molecular mechanism studies showed that LINC00641 suppressed the proliferation and migration of ovarian cancer cells by targeting miR-320a expression, and miR-320a overexpression significantly blocked the inhibitory effect. Therefore, LINC00641/miR-320a axis may be a new target for the early diagnosis, treatment, or prognosis of ovarian cancer patients. 


\section{Acknowledgments}

Funding: This work was supported by the Yancheng Medical Science and Technology Development Plan project in 2020 (No. YK2020010, No. YK20200220).

\section{Footnote}

Reporting Checklist: The authors have completed the MDAR reporting checklist. Available at https://dx.doi. org/10.21037/tcr-21-2314

Data Sharing Statement: Available at https://dx.doi. org/10.21037/tcr-21-2314

Conflicts of Interest: All authors have completed the ICMJE uniform disclosure form (available at https://dx.doi. org/10.21037/tcr-21-2314). The authors have no conflicts of interest to declare.

Ethical Statement: The authors are accountable for all aspects of the work in ensuring that questions related to the accuracy or integrity of any part of the work are appropriately investigated and resolved. This study was approved by the ethics committee of the First People's Hospital of Yancheng (No. 91921626), and the informed consent was signed by the patients or their families. All procedures performed in this study involving human participants were in accordance with the Declaration of Helsinki (as revised in 2013).

Open Access Statement: This is an Open Access article distributed in accordance with the Creative Commons Attribution-NonCommercial-NoDerivs 4.0 International License (CC BY-NC-ND 4.0), which permits the noncommercial replication and distribution of the article with the strict proviso that no changes or edits are made and the original work is properly cited (including links to both the formal publication through the relevant DOI and the license). See: https://creativecommons.org/licenses/by-nc-nd/4.0/.

\section{References}

1. Bonifácio VDB. Ovarian Cancer Biomarkers: Moving Forward in Early Detection. Adv Exp Med Biol 2020;1219:355-63.

2. Menon U, Karpinskyj C, Gentry-Maharaj A. Ovarian Cancer Prevention and Screening. Obstet Gynecol
2018;131:909-27.

3. Yuan Y, Wang R, Guo F, et al. A clinical model to predict the risk of liver metastases in newly diagnosed ovarian cancer: a population-based study. Transl Cancer Res 2020;9:7044-53.

4. Lheureux S, Braunstein M, Oza AM. Epithelial ovarian cancer: Evolution of management in the era of precision medicine. CA Cancer J Clin 2019;69:280-304.

5. Bhan A, Soleimani M, Mandal SS. Long Noncoding RNA and Cancer: A New Paradigm. Cancer Res 2017;77:3965-81.

6. Chan JJ, Tay Y. Noncoding RNA:RNA Regulatory Networks in Cancer. Int J Mol Sci 2018;19:1310.

7. Lorenzi L, Avila Cobos F, Decock A, et al. Long noncoding RNA expression profiling in cancer: Challenges and opportunities. Genes Chromosomes Cancer 2019;58:191-9.

8. Du W, Feng Z, Sun Q. LncRNA LINC00319 accelerates ovarian cancer progression through miR423-5p/NACC1 pathway. Biochem Biophys Res Commun 2018;507:198-202.

9. Liang H, Yu T, Han Y, et al. LncRNA PTAR promotes EMT and invasion-metastasis in serous ovarian cancer by competitively binding miR-101-3p to regulate ZEB1 expression. Mol Cancer 2018;17:119.

10. Shen W, Xie X, Liu M, et al. Diagnostic Value of lncRNA ROR in Differentiating Ovarian Cancer Patients. Clin Lab 2020. doi: 10.7754/Clin.Lab.2019.191035.

11. Li Y, Zhao L, Zhao P, et al. Long non-coding RNA LINC00641 suppresses non-small-cell lung cancer by sponging miR-424-5p to upregulate PLSCR4. Cancer Biomark 2019;26:79-91.

12. Wang J, Liu ZH, Yu LJ. Long non-coding RNA LINC00641 promotes cell growth and migration through modulating miR-378a/ZBTB20 axis in acute myeloid leukemia. Eur Rev Med Pharmacol Sci 2019;23:7498-509.

13. Peng WX, Koirala P, Mo YY. LncRNA-mediated regulation of cell signaling in cancer. Oncogene 2017;36:5661-7.

14. Wang J, Zhu S, Meng N, et al. ncRNA-Encoded Peptides or Proteins and Cancer. Mol Ther 2019;27:1718-25.

15. Tian XJ, Ferro MV, Goetz H. Modeling ncRNA-Mediated Circuits in Cell Fate Decision. Methods Mol Biol 2019;1912:411-26.

16. Jin Y, Feng SJ, Qiu S, et al. LncRNA MALAT1 promotes proliferation and metastasis in epithelial ovarian cancer via the PI3K-AKT pathway. Eur Rev Med Pharmacol Sci 2017;21:3176-84. 
17. Wang $X$, Yang B, She $Y$, et al. The lncRNA TP73-AS1 promotes ovarian cancer cell proliferation and metastasis via modulation of MMP2 and MMP9. J Cell Biochem 2018;119:7790-9.

18. Huang Y. The novel regulatory role of IncRNA-miRNAmRNA axis in cardiovascular diseases. J Cell Mol Med 2018;22:5768-75.

19. Ferrè F, Colantoni A, Helmer-Citterich M. Revealing protein-lncRNA interaction. Brief Bioinform 2016;17:106-16.

20. Hu Y, Su Y, Lei X, et al. LINC00641/miR-582-5p mediate oxaliplatin resistance by activating autophagy in gastric adenocarcinoma. Sci Rep 2020;10:14981.

21. Yang J, Yu D, Liu X, et al. LINC00641/miR-4262/NRGN axis confines cell proliferation in glioma. Cancer Biol Ther 2020;21:758-66.

22. Fu G, Lu J, Zheng Y, et al. MiR-320a inhibits malignant phenotype of melanoma cells via targeting PBX3. J BUON 2020;25:2071-7.

23. Li Y, Liu H, Shao J, et al. miR-320a serves as a negative regulator in the progression of gastric cancer by targeting RAB14. Mol Med Rep 2017;16:2652-8.

24. Kong L, Sun Y, Chen M, et al. Downregulation of microRNA-320a inhibits proliferation and induces apoptosis of retinoblastoma cells via targeting TUSC3. Exp Ther Med 2020;20:9.

(English Language Editor: A. Kassem)
Cite this article as: $\mathrm{Li} \mathrm{Y,} \mathrm{Lv} \mathrm{M,} \mathrm{Wang} \mathrm{J,} \mathrm{Gao} \mathrm{C,} \mathrm{Wu} \mathrm{Y.}$ LINC00641 inhibits the proliferation and invasion of ovarian cancer cells by targeting miR-320a. Transl Cancer Res 2021;10(11):4894-4904. doi: 10.21037/tcr-21-2314 